\title{
HIV-Infection as a Prognostic Factor in Surgical Patients with Non-Small Cell Lung Cancer
}

\author{
Craig M. Hooker, MPH ${ }^{1, *}$, Robert A. Meguid, MD, MPH $2,4,{ }^{*}$, Alicia Hulbert, MD ${ }^{1}$, Joshua T. \\ Taylor, MD ${ }^{2,5}$, James Shin ${ }^{1}$, John Wrangle, MD, MPH ${ }^{1}$, Kristen Rodgers ${ }^{1}$, Beverly Lee ${ }^{1}$, \\ Suvasini Laskshmanan ${ }^{1}$, Travis Brown ${ }^{2}$, Avedis Meneshian, MD², Marc Sussman, MD², \\ Jeanne Keruly, $\mathbf{M S}^{3}$, Richard D. Moore, MD, MHS ${ }^{3}$, Stephen C. Yang, MD ${ }^{1,2}$, and Malcolm V. \\ Brock, MD ${ }^{1,2}$ \\ ${ }^{1}$ Department of Oncology, Johns Hopkins School of Medicine, Baltimore, MD \\ 2 Department of Surgery, Johns Hopkins School of Medicine, Baltimore, MD \\ ${ }^{3}$ Department of Infectious Disease. Johns Hopkins School of Medicine, Baltimore, MD \\ ${ }^{4}$ Department of Surgery, University of Washington, Seattle, WA \\ ${ }^{5}$ Department of Surgery, University of Vanderbilt, Nashville, TN
}

\begin{abstract}
Background-To assess the effect of HIV-infection on postoperative survival among non-small cell lung cancer (NSCLC) patients.

Methods-A retrospective cohort study compared $22 \mathrm{HIV}$-infected lung cancer patients to 2,430 lung cancer patients with HIV-unspecified status resected at Johns Hopkins Hospital from 1985-2009. Sub-cohort comparative analyses were performed using individual matching methods.
\end{abstract}

Results-Thirty day mortality rates did not differ between HIV-infected and HIV-unspecified patients. Survival rates in HIV-infected lung cancer patients were significantly shorter than in HIV-unspecified patients (median 26 vs. 48 months; $p=0.001$ ). After adjustment, the relative hazard of mortality among HIV-infected NSCLC patients was $\geq 3$ fold that of HIV- unspecified patients $(\mathrm{aHR}=3.08,95 \%$ CI $1.85 ; 5.13)$. When additional surgical characteristics were modeled in a matched sub-cohort, the association remained statistically significant (aHR=2.31, 95\% CI 1.11; 4.81). Moreover, HIV-infected lung cancer patients with CD4 counts $<200$ cells $/ \mathrm{mm}^{3}$ had shortened median survival than those with $\mathrm{CD} 4 \geq 200$ cells $/ \mathrm{mm}^{3}$ ( 8 vs. 40 months; $\mathrm{p}=0.031$ ). Postoperative pulmonary and infectious complications were also elevated in the HIV-infected group ( $\mathrm{p}=0.001$ and $<0.001$, respectively). After surgery, median time to cancer progression was shorter among HIV-infected patients (20.4 months) versus HIV-unspecified patients ( $\mathrm{p}=0.061$ ).

Conclusions-HIV-infected NSCLC patients are associated with more postoperative complications, rapid progression to disease recurrence and poorer postoperative survival. Optimizing immune status prior to surgery and careful patient selection based on DLCO may improve patient outcomes.

(C) 2011 The Society of Thoracic Surgeons. Published by Elsevier Inc. All rights reserved.

Corresponding Author: Malcolm V. Brock, M.D., 600 N. Wolfe St., Blalock 240, Johns Hopkins Hospital, Baltimore, MD 21287.

Telephone: 410-614-3891 Fax: 410-614-9428 mabrock@jhmi.edu.

Co-first authors.

Publisher's Disclaimer: This is a PDF file of an unedited manuscript that has been accepted for publication. As a service to our customers we are providing this early version of the manuscript. The manuscript will undergo copyediting, typesetting, and review of the resulting proof before it is published in its final citable form. Please note that during the production process errors may be discovered which could affect the content, and all legal disclaimers that apply to the journal pertain. 


\section{Keywords}

Lung Cancer Surgery; Infection; Outcomes

\section{INTRODUCTION}

Numerous epidemiologic studies have noted an elevated risk of lung cancer among HIVinfected individuals [1-5]. Typically, these patients are younger, have advanced stage, and worse overall survival compared to lung cancer patients from the general population [6-8]. So advanced is their stage at presentation, that only $10-15 \%$ of HIV lung cancer patients undergo curative resection [7]. Due to this paucity of patients with early disease, little data exist on surgical outcomes in these patients [9-18] with a cumulative total in the literature fewer than 25 patients. The present general consensus based on this limited sample size has been to offer surgery with curative intent to HIV-infected NSCLC patients, regardless of their immune state, if there is localized disease and good patient performance status, $[9,16]$.

From 1985 to 2009, we resected 22 HIV-infected NSCLC patients. As increasingly HIVinfected NSCLC patients present for resection, this report examines the clinical efficacy of surgery in this population.

\section{MATERIAL AND METHODS}

\section{Study Population}

HIV infection status of 10,122 lung cancer patients treated between January 1, 1975 and December 31, 2009, was determined by cross-referencing our HIV specialty clinic and SPORE Lung Cancer databases. Consequently, $124 \mathrm{HIV}$-infected lung cancer patients were identified. Since the first HIV-infected lung cancer patient was diagnosed in August 1985, 2,162 patients diagnosed prior to 1985 were excluded. Cancer types other than NSCLC were excluded, which removed 914 patients; 10 of whom were HIV-infected. Of the remaining 7,033 NSCLC patients, 4,581 (65\%) received no surgical intervention, of which 92 were HIV-infected. The final study population comprises 2,452 NSCLC patients who underwent surgery for curative intent, of which 22 were HIV-infected. Johns Hopkins Institutional Review Board approved this study.

\section{Exposure of Interest}

HIV infection was defined as being HIV-serostatus positive in the Johns Hopkins HIV specialty clinic database prior to pathologic confirmed diagnosis of NSCLC. Persons with NSCLC who were not identified as HIV-serostatus positive after cross-referencing the Johns Hopkins HIV specialty clinic and the SPORE Lung Cancer database were classified as HIVunspecified.

\section{Exposure Assessment}

Demographic characteristics, NSCLC subtypes, cancer staging, oncologic treatment, American Anesthesiology Classification morbidity index, HIV/AIDS-related information, and associated risk factors were obtained from the Johns Hopkins SPORE lung cancer database, clinical charts, and the institution's tumor registry using standardized collection forms and quality controlled data entry procedures. Surgical procedures for curative intent were defined as lobectomy, bilobectomy, or pneumonectomy. Sublobar resections were considered curative only when the surgeon documented this intent. 


\section{Outcome Ascertainment}

The primary end point was postoperative survival. Study entry was defined as date of surgery. Patients were followed until date of death, lost to follow-up, or administratively censored five years after resection. Vital status was confirmed with clinical records, death certificates, and the Social Security Death Index. The secondary end point was disease progression after surgery.

\section{Individual Matching}

Since HIV-infected NSCLC patients are younger compared to those without HIV infection [19] and other clinical characteristics related to survival can be disproportionately distributed by HIV status, such as cancer stage, histology, race, sex, NSCLC diagnosis prior to and after HAART era, and even socioeconomic status [7, 8, 16, 19, 20], we attempted to achieve "balance" on the distribution of observed factors that might potentially bias the effect of HIV on survival [21]. Therefore, a representative sub-cohort was drawn from the surgical cohort. Three HIV-unspecified patients were individually matched to each HIVinfected patient by age at diagnosis, sex, race, stage, NSCLC histologic subtype, date of surgery (+/- 5 years), and surgical procedure.

\section{Statistical Analysis}

Comparison of means and medians of continuous variables was performed using the Student's $t$-test (two-sided) and nonparametric Mann-Whitney U test, respectively. Comparisons between proportions for binary and categorical variables were performed using the $\chi^{2}$ test for homogeneity or Fisher's exact test. All hypotheses tests were two-sided, and results were considered statistically significant for $\mathrm{p}$ values $<0.05$.

Survival was illustrated using the Kaplan-Meier method, and the association of covariates with time to death was analyzed using the Cox proportional hazards regression model. In the multivariable models, covariate adjustment for the unmatched surgical cohort was ultimately determined by differences in HIV status on observed characteristics within the entire cohort, prior belief, as well as clinical and biological plausibility. The individual matched subcohort analysis included all the covariates in the unmatched regression model, postoperative complications, and matched covariates. Results of Cox regression are reported as hazard ratios (HR) with 95\% confidence intervals (CIs).

Covariates used for matching methods remained in the multivariable model to account for residual confounding. A test on the basis of Schoenfeld residuals confirmed the proportional hazards assumption for the matched sub-cohort multivariable Cox regression model $(\mathrm{p}=0.993)$. Statistical analyses were performed using STATA 10.0 statistical software (College Station, TX).

\section{RESULTS}

Our NSCLC cohort consisted of 7,033 patients of which 114 HIV-infected NSCLC patients were identified. Among the HIV-infected individuals, 19\% (22/114) underwent surgery for curative intent versus $35 \%(2,430 / 6,919)$ of HIV-unspecified individuals. Comparatively, HIV-infected patients were significantly younger, African-American, and male (Table 1). Both groups were typical of a surgical cohort, each with a similar stage of disease with stage I predominating. Lobectomy was the preferred procedure, and most patients had adenocarcinoma. Likely due to younger age, HIV-infected patients smoked fewer packyears compared to HIV-unspecified patients, 43 versus 54 pack-years, respectively $(\mathrm{p}=0.012)$. HIV-infected patients had a longer delay from diagnosis to surgery. 


\section{Entire Cohort (Unmatched)}

The 30-day mortality among HIV-infected and HIV-unspecified patients in the entire cohort did not differ (Table 1). The postoperative median survival for HIV-infected patients was shorter at 26.4 months vs. 48.4 months for HIV-unspecified patients ( $\mathrm{p}=0.001$; Figure 1). HIV-infection alone conveyed a relative hazard of mortality of more than twice that of HIVunspecified patients (unadjusted hazard ratio $=2.24,95 \% \mathrm{CI}, 1.37,3.68$; Table 2). After adjusting for risk factors related to overall survival among surgical patients into the multivariable Cox regression model, the relative hazard of mortality in the HIV-infected group increased and remained statistically significant [adjusted HR $(\mathrm{aHR})=3.08,95 \% \mathrm{CI}$ $1.85,5.13]$ (Table 2).

\section{Matched Sub-Cohort}

Characteristics of the individual-matched sub-cohort analysis according to HIV infection status are presented in Table 3a. Balance was achieved on all matched characteristics in the sub-cohort. Fifty-three percent of HIV-unspecified individuals were married compared to $18 \%$ of HIV-infected individuals ( $\mathrm{p}=0.004$ ). In the HIV-unspecified group, the median household income was slightly higher $(\$ 32,458$ vs. $\$ 28,945, \mathrm{p}=0.14)$. Both groups reside mainly within Baltimore City. It is noteworthy that four patients with stage IV disease underwent curative resection, specifically $3 \mathrm{HIV}$-unspecified patients with solitary brain lesions and $1 \mathrm{HIV}$-infected patient with contralateral pulmonary lesions.

The median duration from diagnosis to surgery was longer for HIV-infected patients (72 vs. 40 days; $p=0.02$ ) (table $3 b$ ). HIV-infected patients also experienced longer hospital stays ( 9 vs. 5 days; $\mathrm{p}=0.03$ ); a likely consequence of more post-operative complications. Nearly half of HIV-infected patients experienced $\geq 2$ complications ( $46 \%$ vs. $9 \%, \mathrm{p}<0.001$ ). There were no statistical differences between mean percent predicted forced vital capacity, or mean percent predicted forced expiratory volume in one second. Although mean percent predicted diffusion lung capacity for carbon monoxide (DLCO) differed by HIV status, data were available on only 55\% (12/22) of HIV-infected and 65\% (43/66) of HIV-unspecified patients.

The proportion of adjuvant therapy received was comparable in both groups (Table $3 b$ ). Seventy-six percent of patients with stage II or greater had progressive disease. Patients with stage I compared to stage II or greater experienced similar postoperative complication rates (data not shown). Thirty day mortality did not differ between the two groups (Table 3b). The proportion of lung cancer-related deaths was identical in both groups (Table $3 b$ ).

Individual-matched modeling estimated the adjusted hazard ratios between HIV-infection status and postoperative mortality (Table 4). Matched on age, sex, race, histology, stage, surgical procedure, and surgical date, the crude relative hazard of mortality in the HIVinfected patients increased by $81 \%$ compared to HIV-unspecified patients (crude HR=1.81, 95\% CI 1.02, 3.25; $\mathrm{p}=0.047$ ). In the adjusted model, HIV-infected patients were associated with a significantly higher hazard of mortality relative to HIV-unspecified patients ( $\mathrm{aHR}=2.31 ; 95 \% \mathrm{CI} 1.11,4.81 ; \mathrm{p}=0.026$ ). Having $\geq 2$ complications was associated with an adjusted relative hazard of mortality almost 4 times greater than fewer complications (aHR=3.85; 95\% CI 1.39;10.68; $\mathrm{p}=0.009)$. The cancer-specific survival of the $22 \mathrm{HIV}$ infected surgical patients differed significantly by CD4 cell count (Figure 2). Survival was significantly shorter among individuals with CD4 cell counts below 200 cells $/ \mathrm{mm}^{3}$ (median survival 8.3 vs. 40.0 months; $\mathrm{p}=0.031$ ). Of the 6 patients with CD4 count $<200$ cells $/ \mathrm{mm}^{3}$, five died. Four patients died from lung cancer, and one patient from AIDS-related causes. Four of the six patients with CD4 counts $<200$ also had cancer progression after surgery. Additionally, median time after surgery to cancer progression was shorter among HIV- 
infected compared to HIV-unspecified patients (20.4 months vs. not yet reaching $50^{\text {th }}$ percentile, $\mathrm{p}=0.061$, Figure 3 ).

\section{COMMENT}

Compared to HIV-unspecified NSCLC patients, HIV-infected NSCLC patients displayed grim postoperative survival. Overall and progression-free survival is equally dismal among HIV-infected patients. Even after adjustment for important clinical prognostic indicators, HIV-infected patients exhibited greater mortality. Furthermore, immunosuppression at surgery correlates with rapid decline in survival. This finding runs contrary to prior recommendations advocating surgery for HIV-infected patients regardless of immune status [16].

Given the strong relationship between stage and survival, it was expected that stage would account for the majority of variance in the model and attenuate the effect of HIV-infected status [7, 22]. Instead, after matching and conditioning on stage, the effect of HIV infection increased, indicating that even with similar NSCLC stage, HIV-infected individuals have significantly poorer survival postoperatively. To account for stage migration over the study's 20 years, the surgical year was included in our regression models and matched study design. Nonetheless, the impact of HIV on survival in either the entire analytic cohort or in the matched sub-cohort estimates remained elevated. Compared to HIV-unspecified patients, HIV-infected individuals underwent surgery a month later after diagnosis. We speculate that this difference is indicative of potential barriers to medical care access commonly associated with HIV-infected populations, including low income, lack of medical insurance, HIVrelated co-morbidities, drug abuse, and even transient residence [23]. Nevertheless, any delay in surgery had no detrimental effect on survival in HIV patients on multivariable analysis.

We show differences in survival between HIV patients by CD4 cell count, corroborating Thurer et al. who found in $4 \mathrm{HIV}$-positive NSCLC surgical patients, long-term survival in the sole patient with CD4 lymphocytes $>200$ cells $/ \mathrm{mm}^{3}$ [11]. This value is not completely arbitrary since constitutional symptoms begin in HIV patients with CD4 counts $<300$ cells/ $\mathrm{mm}^{3}$, serious opportunistic infections occur at CD4 counts $<100 \mathrm{cells} / \mathrm{mm}^{3}$, [11] and from 1992 to 2006, the Center for Disease Control defined AIDS as CD4 cell counts < 200 cells/ $\mathrm{mm}^{3}$ [24]. In this study, the CD4 cell count was measured as a fixed variable at the closest time prior to surgery. CD4 count can vary markedly within an individual, especially when patients are non-compliant with antiretroviral regimens [25]. Multiple measures of CD4 cell counts or of the nadir CD4 cell counts could reveal persistently low values that may better characterize immunosuppression.

In our study, HIV-infected patients were significantly more likely to develop $\geq 2$ postoperative complications than HIV-unspecified patients. Increased postoperative complications, in general, and postoperative pulmonary complications, in particular, were associated with decreased pulmonary functional status [26, 27]. Although DLCO might be a relevant factor, we hesitate to make definitive conclusions due to few study patients with DLCO data. HIV-infected patients also showed increased progression to recurrence. This maybe important since it suggests a potential biologic mechanism of NSCLC progression involving immunosuppression [28].

The obvious limitation of this study is the lack of precision inherent in a small sample size precluding strong recommendations. The observed estimates of effect could simply be due to random variability. Second, it was uncommon to test for HIV antibodies during the initial work-up of the general NSCLC patient at our institution. It is plausible, albeit unlikely, that 
at NSCLC diagnosis, some individuals with sub-clinical HIV-infection could have been misclassified. Lastly, the treatment assignment of HIV infection status is a complex construct of many factors related to health and survival. The mechanism through which decreased survival is associated with HIV infection is not fully understood within the context of this study. Notwithstanding, this study utilized a powerful design and analytic methods to improve statistical efficiency and balance on HIV infection status. Unmeasured covariates that could account for the observed effect would have to be strongly associated with HIV infection and survival.

Despite HIV-infected patients having comparable 30-day mortality rates to HIV-unspecified patients, surgery is associated with more postoperative complications, rapid progression to recurrence and poorer survival rates. Shortened progression-free and overall survival seem particularly evident in patients with chronically suppressed immune status, i.e. patients with CD4 counts $<200$ cells $/ \mathrm{mm}^{3}$. Careful HIV-infected patient selection based on DLCO may improve postoperative complication rates, and optimizing immune status preoperatively may ameliorate cancer survival rates, but the lack of statistical power precludes definitive recommendations. Future investigations should consider pooled analytic designs as well as prospective measures of HIV immunosuppression and DLCO.

\section{Acknowledgments}

We sincerely thank Kathryn Bender and Josephine Allen for their assistance in manuscript preparation; sponsors include the NIH P50 CA058184, P30-A142855, and 5K23CA117820-03.

\section{References}

1. Chaturvedi AK, et al. Elevated risk of lung cancer among people with AIDS. Aids. 2007; 21(2): 207-13. [PubMed: 17197812]

2. Engels EA, et al. Elevated incidence of lung cancer among HIV-infected individuals. J Clin Oncol. 2006; 24(9):1383-8. [PubMed: 16549832]

3. Dal Maso L, et al. Lung cancer in persons with AIDS in Italy, 1985-1998. Aids. 2003; 17(14):21179. [PubMed: 14502015]

4. Shiels MS, et al. Lung cancer incidence and mortality among HIV-infected and HIV-uninfected injection drug users. J Acquir Immune Defic Syndr. 55(4):510-5. [PubMed: 20838223]

5. Kirk GD, et al. HIV infection is associated with an increased risk for lung cancer, independent of smoking. Clin Infect Dis. 2007; 45(1):103-10. [PubMed: 17554710]

6. Powles T, et al. Does HIV adversely influence the outcome in advanced non-small-cell lung cancer in the era of HAART? Br J Cancer. 2003; 89(3):457-9. [PubMed: 12888811]

7. Brock MV, et al. Delayed diagnosis and elevated mortality in an urban population with HIV and lung cancer: implications for patient care. J Acquir Immune Defic Syndr. 2006; 43(1):47-55. [PubMed: 16936558]

8. Bower M, et al. HIV-related lung cancer in the era of highly active antiretroviral therapy. Aids. 2003; 17(3):371-5. [PubMed: 12556691]

9. Spano JP, et al. Lung cancer in patients with HIV Infection and review of the literature. Med Oncol. 2004; 21(2):109-15. [PubMed: 15299182]

10. Vyzula R, Remick SC. Lung cancer in patients with HIV-infection. Lung Cancer. 1996; 15(3): 325-39. [PubMed: 8959678]

11. Thurer RJ, et al. Surgical treatment of lung cancer in patients with human immunodeficiency virus. Ann Thorac Surg. 1995; 60(3):599-602. [PubMed: 7677486]

12. Mouroux J, et al. Surgical management of thoracic manifestations in human immunodeficiency virus-positive patients: indications and results. Br J Surg. 1995; 82(1):39-43. [PubMed: 7881953]

13. Sridhar KS, et al. Lung cancer in patients with human immunodeficiency virus infection compared with historic control subjects. Chest. 1992; 102(6):1704-8. [PubMed: 1446476] 
14. Massera F, et al. Pulmonary resection for lung cancer in HIV-positive patients with low ( $<200$ lymphocytes/mm(3)) CD4(+) count. Lung Cancer. 2000; 29(2):147-9. [PubMed: 10963845]

15. Lavole A, et al. Lung cancer, a new challenge in the HIV-infected population. Lung Cancer. 2006; 51(1):1-11. [PubMed: 16300854]

16. Cadranel J, et al. Lung cancer in HIV infected patients: facts, questions and challenges. Thorax. 2006; 61(11):1000-8. [PubMed: 17071836]

17. Bazot M, et al. Computed tomographic diagnosis of bronchogenic carcinoma in HIV-infected patients. Lung Cancer. 2000; 28(3):203-9. [PubMed: 10812189]

18. Aviram G, Fishman JE, Schwartz DS. Metachronous primary carcinomas of the lung in an HIVinfected patient. AIDS Patient Care STDS. 2001; 15(6):297-300. [PubMed: 11445011]

19. Shiels MS, Pfeiffer RM, Engels EA. Age at cancer diagnosis among persons with AIDS in the United States. Ann Intern Med. 153(7):452-60. [PubMed: 20921544]

20. Ou SH, et al. Low socioeconomic status is a poor prognostic factor for survival in stage I nonsmall cell lung cancer and is independent of surgical treatment, race, and marital status. Cancer. 2008; 112(9):2011-20. [PubMed: 18361399]

21. Stuart EA. Matching methods for causal inference: A review and a look forward. Stat Sci. 25(1):121. [PubMed: 20871802]

22. Meguid RA, et al. Long-term survival outcomes by smoking status in surgical and nonsurgical patients with non-small cell lung cancer: comparing never smokers and current smokers. Chest. 138(3):500-9. [PubMed: 20507946]

23. Krawczyk CS, et al. Factors associated with delayed initiation of HIV medical care among infected persons attending a southern HIV/AIDS clinic. South Med J. 2006; 99(5):472-81. [PubMed: 16711309]

24. 1993 revised classification system for HIV infection and expanded surveillance case definition for AIDS among adolescents and adults. MMWR Recomm Rep. 1992; 41(RR-17):1-19.

25. Malta M, et al. Adherence to antiretroviral therapy for human immunodeficiency virus/acquired immune deficiency syndrome among drug users: a systematic review. Addiction. 2008; 103(8): 1242-57. [PubMed: 18855813]

26. Ferguson MK, Vigneswaran WT. Diffusing capacity predicts morbidity after lung resection in patients without obstructive lung disease. The Annals Of Thoracic Surgery. 2008; 85(4):11581164. [PubMed: 18355489]

27. Cerfolio RJ, Bryant AS. Different diffusing capacity of the lung for carbon monoxide as predictors of respiratory morbidity. The Annals Of Thoracic Surgery. 2009; 88(2):405-410. [PubMed: 19632384]

28. Wistuba II, et al. Comparison of molecular changes in lung cancers in HIV-positive and HIVindeterminate subjects. Jama. 1998; 279(19):1554-9. [PubMed: 9605900] 


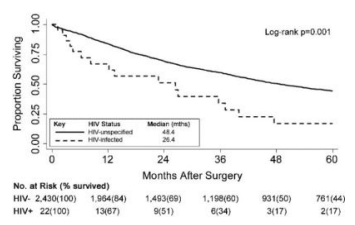

Figure 1.

Kaplan-Meier curves of all-cause survival following surgery comparing HIV-unspecified $(\mathrm{n}=2,430)$ to HIV-infected $(\mathrm{n}=22)$ NSCLC patients. 


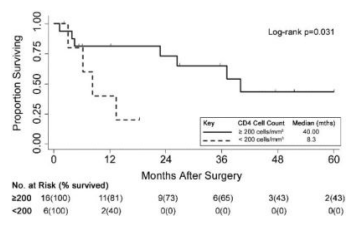

Figure 2.

Kaplan-Meier curves of cancer-specific survival following surgery for HIV-infected NSCLC patients $(\mathrm{n}=22)$ by CD4 cell counts, $<200$ cells $/ \mathrm{mm}^{3}(\mathrm{n}=6)$ vs. $\geq 200 \mathrm{cells} / \mathrm{mm}^{3}(\mathrm{n}=16)$. 


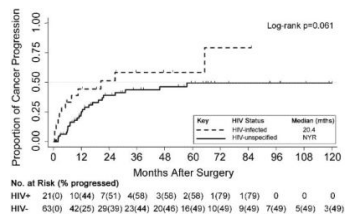

Figure 3.

Cumulative probability of cancer progression post-surgery comparing matched HIVunspecified $(\mathrm{n}=63)$ to HIV-infected $(\mathrm{n}=21)$ NSCLC patients. * Stage IV patients excluded (4); NYR, not yet reached $50^{\text {th }}$ percentile. 


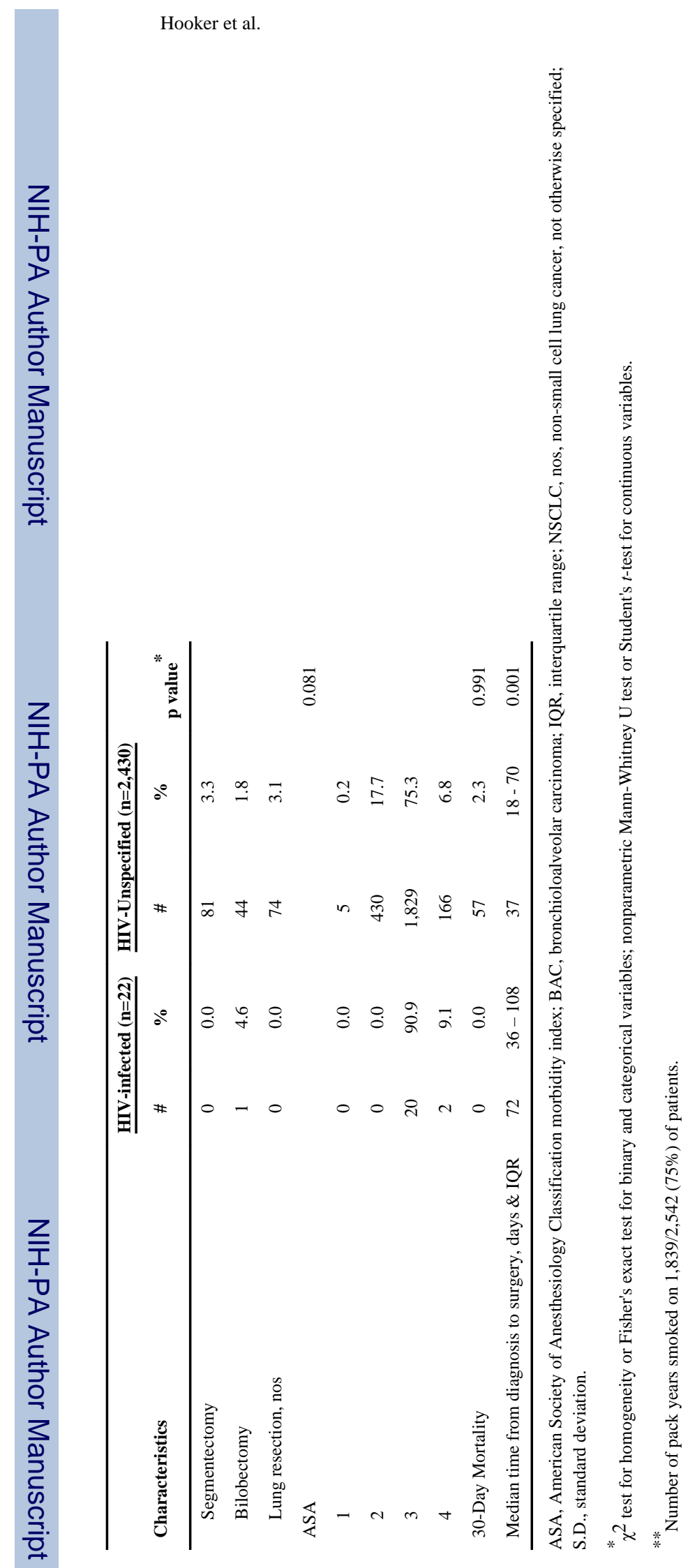

Ann Thorac Surg. Author manuscript; available in PMC 2013 February 1. 


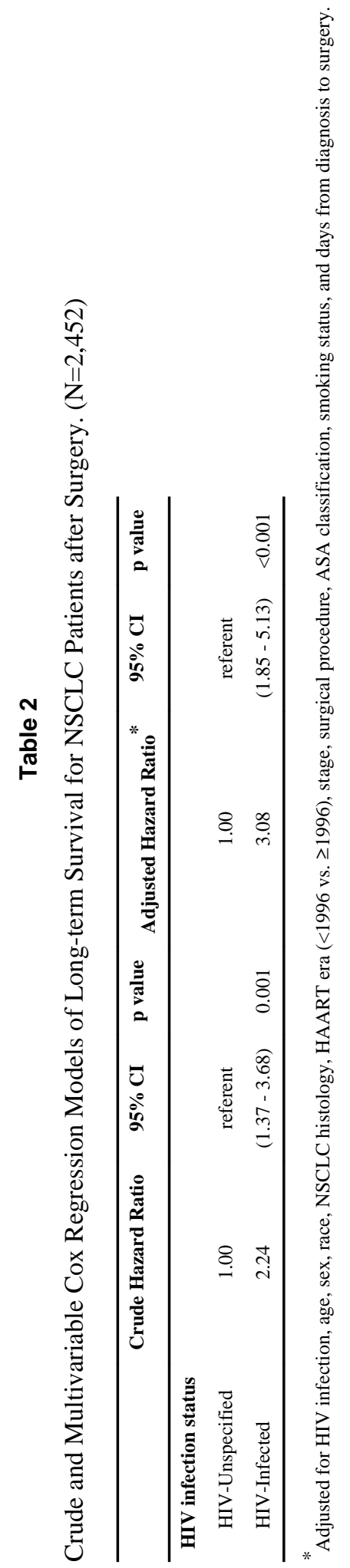

Ann Thorac Surg. Author manuscript; available in PMC 2013 February 1. 


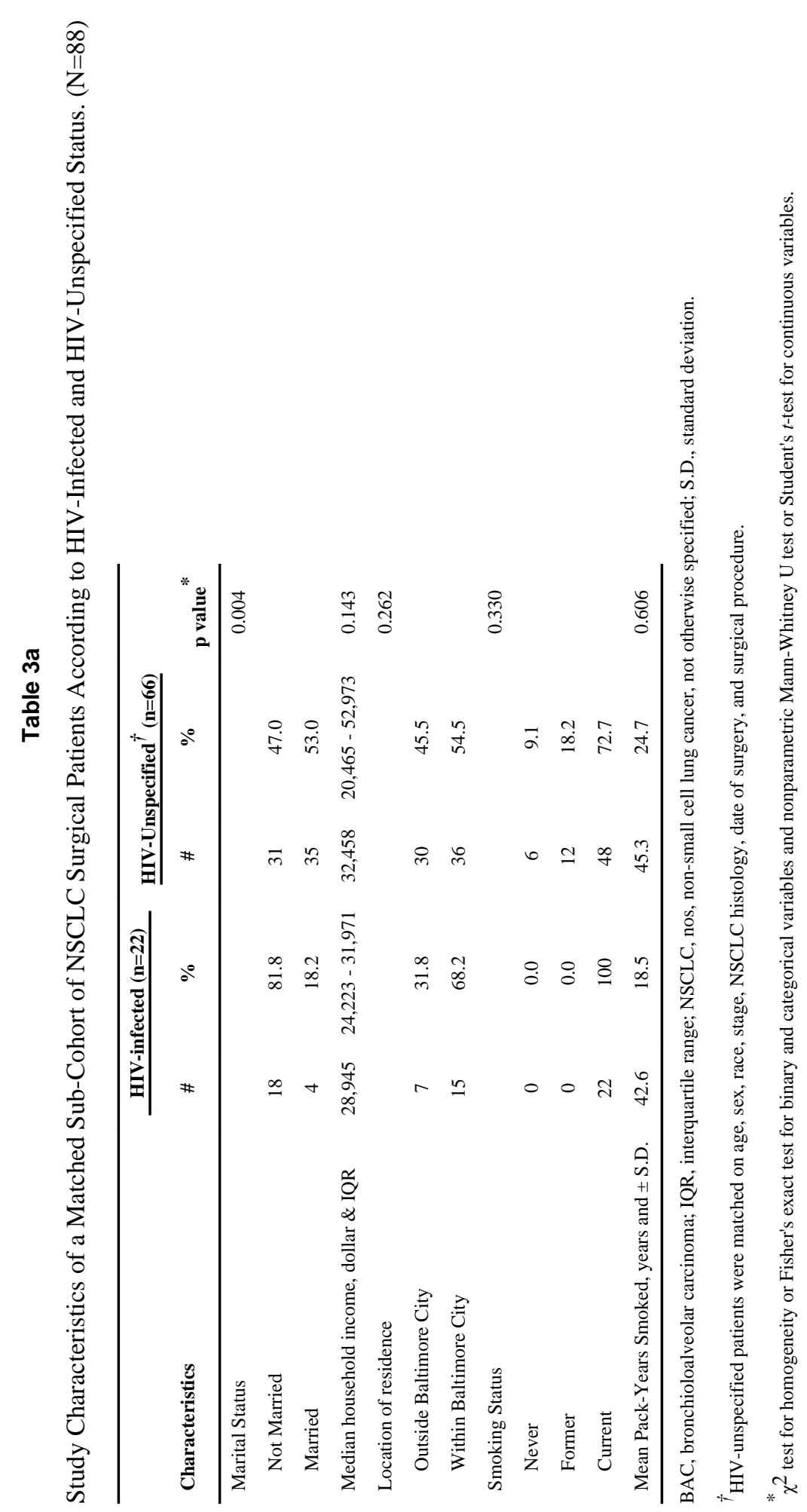




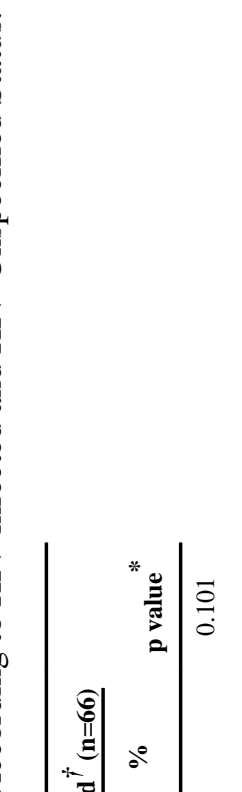

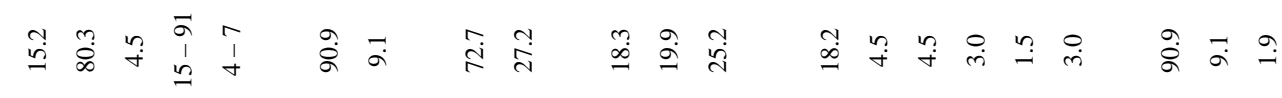




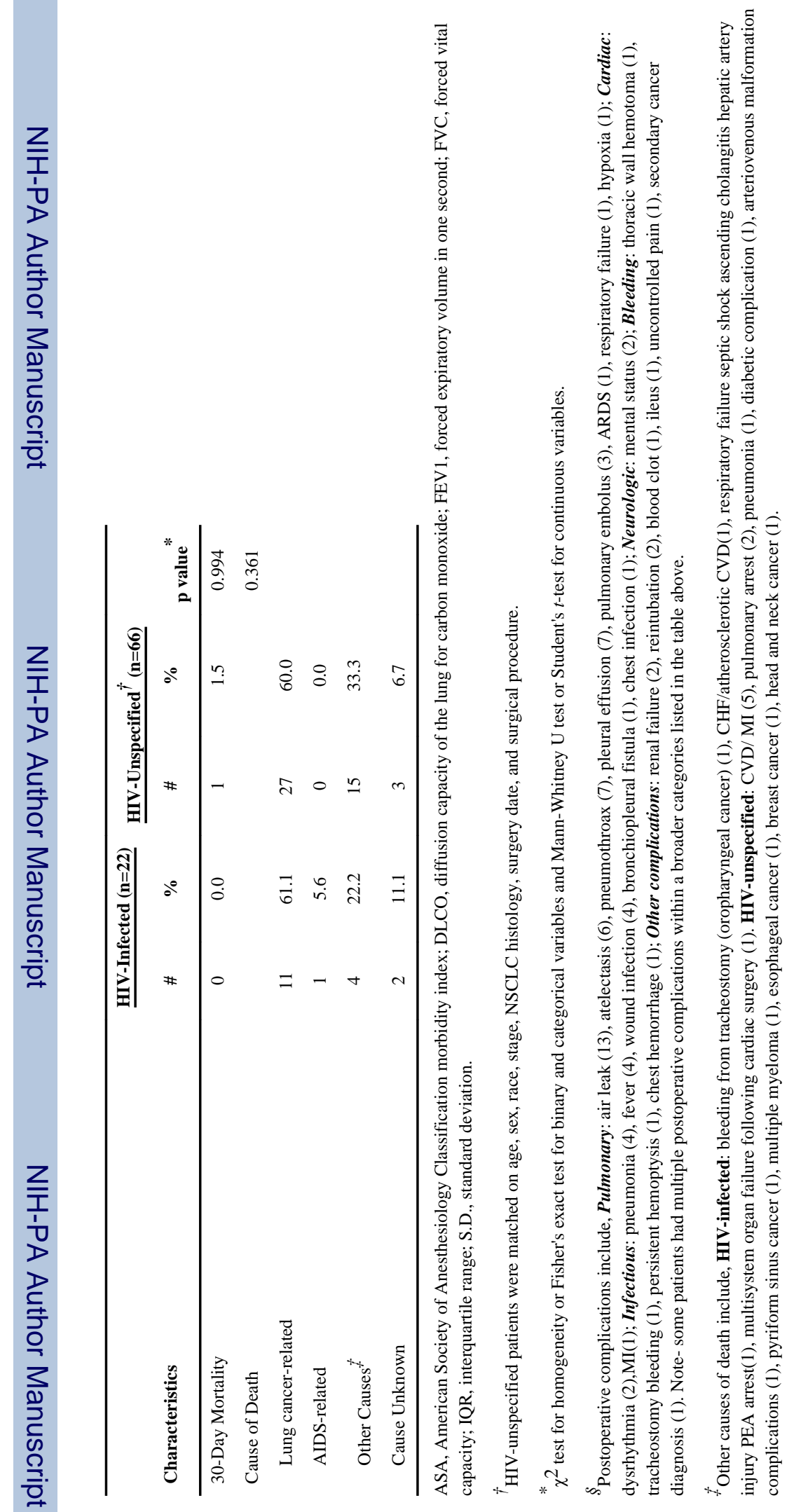




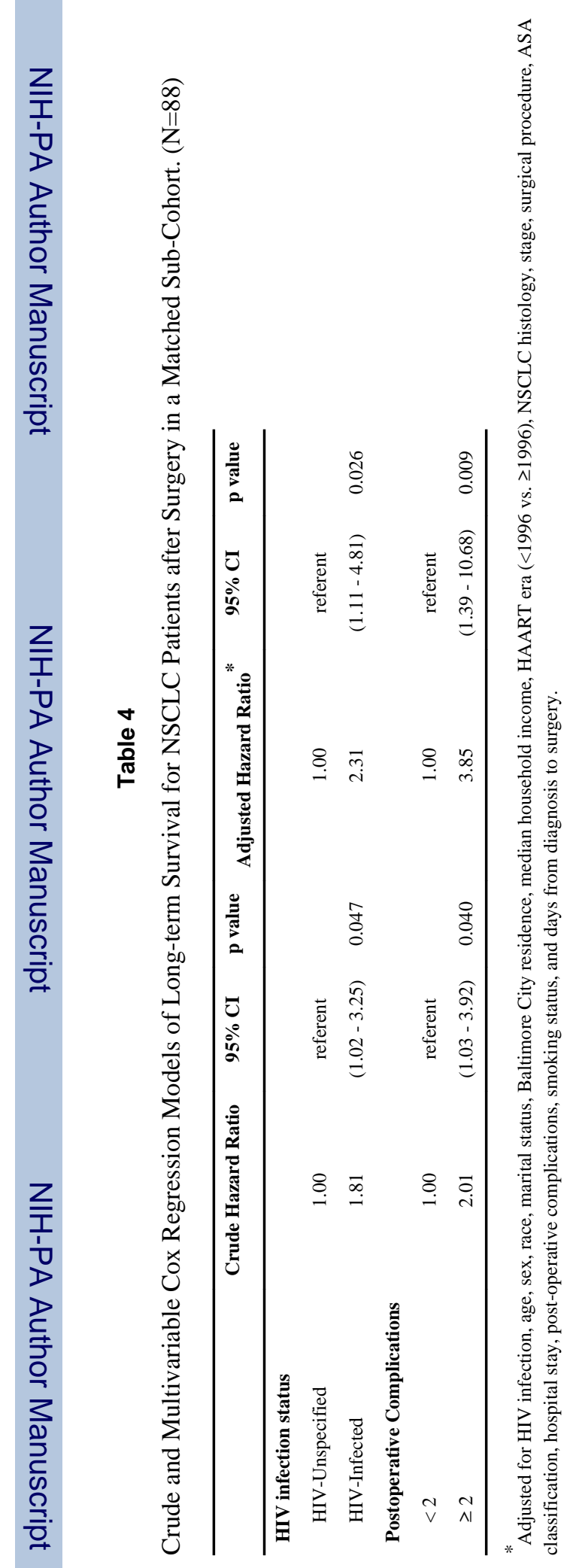

Ann Thorac Surg. Author manuscript; available in PMC 2013 February 1. 